\title{
Subtipos clínicos y genéticos de cáncer de mama: individualización del tratamiento
}

Khosravi Shahi P, Pérez Manga G. Subtipos clínicos y genéticos de cáncer de mama: individualización del tratamiento. An Med Interna (Madrid) 2007; 24: 569-570.

El cáncer de mama invasivo (CMI) es el tumor maligno más frecuente en la mujer en los países occidentales. Más del 90\% de los CMI son esporádicos, y tan sólo el 10-15\% son de carácter hereditario o familiar (generalmente asociado a mutaciones de los genes BRCA 1 y 2, y TP53) (1).

El CMI constituye un grupo heterogéneo de neoplasias, que presentan una historia natural diferente. Los estudios más recientes mediante el empleo de la tecnología de microarrays del DNA, que permite el estudio sincrónico de miles de genes expresados por un mismo tumor, ponen de manifiesto la existencia de al menos tres grandes subtipos de CMI desde el punto de vista del perfil genético, con relevancia pronostica y terapéutica (2):

-El CMI tipo Luminal: Este subtipo genotípico de CMI se caracteriza por una asociación con una alta expresión de receptores estrogénicos (RE) y a los genes regulados por los estrógenos, lo que proporciona una alta tasa de respuesta a la hormonoterapia. Además este subtipo se asocia a un mejor pronostico.

-El CMI HER-2 positivo: Esta variante presenta el genotipo de RE negativos y se asocia con la sobreexpresión de genes relacionados con una mayor agresividad biológica, lo que conlleva un peor pronostico. Este subtipo presenta una resistencia relativa a determinados agentes quimioterápicos, como el esquema clásico CMF (Ciclofosfamida, Metotrexate y Fluoruracilo) y al tamoxifeno (3). Sin embargo, su historia natural ha sufrido un cambio con la introducción en el mercado del anticuerpo monoclonal anti-HER-2, denominado trastuzumab (4).

-El CMI "Basal-like": Este subtipo de CMI también es conocido como el fenotipo triple negativo (FTN), puesto que suele presentar negatividad para los RE, receptores progesterónicos (RP) y receptores HER-2. En un 50\% de los casos los carcinomas de mama "Basal-like" presentan una sobreexpresión del receptor de factor de crecimiento epidérmico (EGFR), y generalmente se asocian con hallazgos histopatológicos de mal pronostico como alto grado nuclear, pleomorfismo o un alto índice mitótico. Por tanto, se trata de un subtipo de CMI de mal pronóstico, pero que paradójicamente se asocia a una alta tasa de respuesta a la quimioterapia. Sin embar- go, no existe una exacta asociación entre el fenotipo inmunohistoquímico triple negativo (RE, RP y HER-2 negativos por inmunohistoquímica) y el perfil genético "Basal-like".

La opción de curación del CMI pasa por un diagnóstico precoz, que a su vez debe conllevar una actitud terapéutica óptima, que aumente las opciones de control a largo plazo. De esta manera, la cirugía continúa teniendo un papel clave en el tratamiento radical del CMI, aunque su correcto tratamiento requiere un manejo multidisciplinario. La quimioterapia adyuvante (postoperatoria) está indicado en los casos de CMI completamente resecados, que cumplan criterios de alto riesgo de recidiva. Existen dos grandes grupo de citostáticos que han demostrado claramente su eficacia en el tratamiento complementario de CMI: las antraciclinas (adriamicina y epirrubicina) y los taxanos (paclitaxel y docetaxel). El meta-análisis publicado por Early Breast Cancer Trialists' Collaborative Group (EBCTCG) (5), demostró que la quimioterapia adyuvante de unos 6 meses de duración con esquemas basados en antraciclinas, reduce el riesgo anual de muerte por CMI en $38 \%$ en las mujeres menores de 50 años, y en casi $20 \%$ en mujeres con edades comprendidas entre los 50 y 69 años. Además demostró que las antraciclinas disminuyen significativamente tanto la probabilidad de recidiva, como el riesgo de muerte por cáncer cuando se compara con el esquema clásico de CMF ( $\mathrm{p}=0,0001, \mathrm{y} \mathrm{p}=0,00001$; respectivamente). Los taxanos también han demostrado su eficacia en la adyuvancia de CMI, sobre todo en el subgrupo con afectación ganglionar, que presentan un mayor riesgo de recidiva. En estos casos la asociación de los taxanos a las antraciclinas mejora claramente la supervivencia libre de enfermedad (SLE) y la supervivencia global (SG) como lo evidencia los siguientes estudios: a) El estudio encabezado por Miguel Martín (6) (fase III), con 1491 pacientes demostró la superioridad del esquema TAC (Docetaxel, Adriamicina y Ciclofosfamida) sobre el esquema FAC (Fluoruracilo, Adriamicina y Ciclofosfamida), en la adyuvancia de CMI. Con una mediana de seguimiento de 55 meses la probabilidad de SLE a los 5 años era del $75 \%$ en la rama de TAC, frente al $68 \%$ de la rama de FAC $(\mathrm{p}=0,001)$. Así mismo, en el grupo del TAC hubo una reducción del $30 \%$ en el riesgo de muerte $(p=0,008)$; $b)$ El trabajo llevado a cabo 
por Bria y colaboradores (7), con más de 15.500 pacientes con CMI, demostró un incremento estadísticamente significativo a favor de los taxanos en la SLE en el conjunto global de los pacientes ["Hazard Ratio" (HR) = 0,86; p < 0,00001], así como en el subgrupo con ganglios positivos (HR: 0,84; $\mathrm{P}<0.0001)$. También se obtuvo un beneficio en la SG tanto en el conjunto total (HR: 0,87; p < 0.0001), como en la población con afectación ganglionar (HR: 0,84; p < 0,0001); y c). El estudio NSABP B-28 (8) evidenció que el tratamiento secuencial de antraciclinas y taxanos conseguía aumentar significativamente la SLE (HR = 0,83; IC95\%: 0,72-0,95; $p=0,006)$, pero no la $\mathrm{SG}(\mathrm{HR}=0,93 ; \mathrm{p}=0,46)$ en las pacientes con ganglios positivos.

Por otra parte, la eficacia de los taxanos en las pacientes con CMI con ganglios negativos de alto riesgo, está siendo investigado. Un reciente estudio demuestra la superioridad del nuevo esquema TC (Docetaxel y Ciclofosfamida) sobre el esquema clásico AC (Adriamicina y Ciclofosfamida), con independencia de afectación o no de los ganglios (9).

Una nueva estrategia terapéutica en la lucha contra las neoplasias es la inhibición de la angiogenésis asociada al cáncer (10). Las células tumorales en el cáncer de mama pueden llegar a producir hasta 6 tipos diferentes de proteínas proangiogénicas: VEGF, bFGF, TGF $\beta-1$, PLGF, PD-ECGF y pleiotrofina. Existen diferentes mecanismos de inhibición de

\section{Bibliografía}

1. Ford D, Easton DF, Stratton M. Genetic heterogeneity and penetrance analysis of the BRCA1 and BRCA2 genes in breast cancer families. The breast cancer linkage consortium. Am J Hum Genet 1998; 62: 676-81.

2. Khosravi P, Pérez-Manga G. La aplicación de la tecnología de los Microarrays en la Oncología clínica. An Med Interna (Madrid) 2006; 23: 255-6.

3. Khosravi P, Pérez-Manga G. La relevancia clínica de la sobreexpresión de HER-2 en el cáncer de mama. An MED Interna 2006;23:103-4.

4. Vogel CL, Cobleigh MA, Tripathy D, Gutheil JC, Harris LN, Fehrenbacher L, et al. Efficacy and safety of trastuzumab as a single agent in first-line treatment of HER2-overexpressing metastatic breast cancer. J Clin Oncol 2002; 20: 719-26.

5. Early Breast Cancer Trialists' Collaborative Group (EBCTCG). Effects of chemotherapy and hormonal therapy for early breast cancer on recurrence and 15-year survival: An overview of the randomised trials. Lancet 2005; 365: 1687-717.

6. Martin M, Pienkowski T, Mackey J, et al. Adjuvant docetaxel for nodepositive breast cancer. N Engl J Med 2005; 352: 2302-13. la angiogenésis tumoral como puede ser el empleo de bevacizumab (anticuerpo monoclonal anti-VEGF), los inhibidores tirosin-kinasas multidiana (sunitinib,sorafenib) o la quimioterapia metronómica.

En la actualidad existe una tendencia a individualizar el tratamiento para cada subgrupo clínico de CMI. De esta manera, el subgrupo de CMI hormonosensible (con RE y/o RP positivos) se benefician de la hormonoterapia adyuvante, tras completar la quimioterapia y/o radioterapia según cada caso (11); mientras que el subgrupo con sobreexpresión de HER-2 se beneficia de trastuzumab (12). Sin embargo, en el momento actual no existen recomendaciones a cerca del tratamiento complementario en el subgrupo de FTN. Un nuevo ensayo clínico pretende estudiar la eficacia del tratamiento antiangiogénico en la adyuvancia de este subgrupo de CMI de mal pronóstico.

\section{P. KHOSRAVI SHAHI, G. PÉREZ MANGA}

\author{
Hospital General Universitario "Gregorio Marañón”. \\ Servicio de Oncología Médica. Madrid
}

7. Bria E, Nistico C, Cuppone F, et al. Benefit of taxanes as adjuvant chemotherapy for early breast cancer: Pooled analysis of 15,500 patients. Cancer 2006; 106: 2337-44.

8. Mamounas EP, Bryant J, Lembersky B, et al. Paclitaxel after doxorubicin plus cyclophosphamide as adjuvant chemotherapy for node-positive breast cancer: Results from NSABP B-28. J Clin Oncol 2005; 23: 368696.

9. Jones SE, Savin MA, Holmes FA, et al. Phase III trial comparing Doxorubicin plus Cyclophosphamide with Docetaxel plus Cyclophosphamide as Adjuvant Therapy for Operable Breast Cancer. J Clin Oncol 2006; 24: 5381-7.

10. Khosravi P. Angiogénesis y neoplasias. An Med Interna (Madrid) 2006; 23: 355-6.

11. Pritchard KI. The best use of adjuvant endocrine treatments. Breast 2003; 12: 497-503.

12. Romond EH, Pérez EA, Bryant J, et al. Trastuzumab plus adjuvant chemotherapy for operable HER2-positive breast cancer. N Engl J Med 2005; 353: 1673-84. 